\title{
Human Factor Analysis about Human Error on Aviation Maintenance
}

\author{
Rong Cheng \\ Guanghan Sub-college, \\ Civil Aviation Flight University of China, \\ Guanghan, Sichuan, 618307, China \\ sancer123@163.com
}

\begin{abstract}
The purpose of this study was to analysis the human factors that contribute to maintenance errors and making corrective actions to eliminate or reduce the probability of future, similar errors. With the help of the human factor theory, through deep analysis and research to maintenance-related aviation accidents and incidents caused by human error, twelve human factors could lead to maintenance errors. Through training and study, AMT (aviation maintenance technician) can understand the interaction between organizational, work group, and individual factors that may lead to errors and accidents, AMTs can learn to prevent or manage them proactively in the future. The research like this is valuable because maintenance errors have safe ty and economic cons equences to the airline industry[1].
\end{abstract}

Keywords-error; maintenance; communication; fatigue; stress; resource; norms

\section{INTRODUCTION}

Human error is defined as a human action with unintended consequences[3]. There is nothing inherently wrong or troublesome with error itself, but when you couple error with aviation maintenance and the negative consequences that it produces, it becomes extremely troublesome. Training, risk assessments, safety inspections, etc., should not be restricted to attempt to avoid errors but rather to make them visible and identify them before they produce damaging and regrettable consequences. Simply put, human error is not avoidable but it is manageable.

This paper presents twelve human factors about human error on aviation maintenance. And gives correct actions on how to prevent or manage human factors about aviation maintenance error to AMT.

\section{TYPES OF ERRORS}

\section{A. Unintentional}

An unintentional error is an unintentional wandering or deviation from accuracy. This can include an error in your action (a slip), opinion, or judgment caused by poor reasoning, carelessness, or insufficient knowledge (a mistake). For example, an AMT reads the torque values from a job card and unintentionally transposed the number 96 to 69 . He or she did not mean to make that error but unknowingly and unintentionally did. An example of an unintentional mistake would be selecting the wrong work card to conduct a specific repair or task. Again, not an intentional mistake but a mistake nonetheless.

\section{B. Intentional}

In aviation maintenance, an intentional error should really be considered a violation. If someone knowingly or intentionally chooses to do something wrong, it is a violation, which means that one has deviated from safe practices, procedures, standards, or regulations.

\section{KINDS OF ERRORS}

\section{A. Active and Latent}

An active error is the specific individual activity that is an obvious event. A latent error is the organization issues that lead up to the event. For example, an AMT climbs up a ladder to do a repair knowing that the ladder is broken. In this example, the active error was falling from the ladder. The latent error was the broken ladder that someone should have replaced.

\section{HUMAN FACTORS ANALYSIS ON MAINTENANCE ERROR}

Due to a large number of maintenance-related aviation accidents and incidents that occurred in the late 1980s and early 1990s[7], twelve human factors degrade people's ability to perform effectively and safely, which could lead to maintenance errors[2]. It is important to know these human factors, how to recognize their symptoms, and most importantly, know how to avoid or contain errors produced by them. Understanding the interaction between organizational, work group, and individual factors that may lead to errors and accidents, AMTs can learn to prevent or manage them proactively in the future.

\section{A. Lack of Communication}

Lack of communication is a key human factor that can result in suboptimal, incorrect, or faulty maintenance. Communication occurs between the AMT and many people (i.e., management, pilots, parts suppliers, aircraft servicers). Each exchange holds the potential for misunderstanding or omission. But communication between AMTs may be the most important of all. Lack of communication between technicians could lead to a maintenance error and result in an aircraft accident. This is especially true during procedures where more than one technician performs the work on the aircraft. It is critical that accurate, complete information be exchanged to ensure that all work is completed without any step being omitted. Knowledge and speculation about a task must be 
clarified and not confused. Each step of the maintenance procedure must be performed according to approved instructions as though only a single technician did the work[3].

\section{B. Complacency}

Complacency is a human factor in aviation maintenance that typically develops over time. As a technician gains knowledge and experience, a sense of self satisfaction and false confidence may occur. A repetitive task, especially an inspection item, may be overlooked or skipped because the technician has performed the task a number of times without ever finding a fault. The false assumption that inspection of the item is not important may be made. However, even if rare, a fault may exist. The consequences of the fault not being detected and corrected could cause an incident or accident. Routine tasks performed over and over allow time for the technician's mind to wander, which may also result in a required task not being performed.

To combat complacency, a technician must train oneself to expect to find the fault that created the inspection item in the first place. He or she must stay mentally engaged in the task being performed. All inspection items must be treated with equal importance, and it must never be assumed that an item is acceptable when it has not been inspected. A technician should never sign for any work that has not been performed. Prior to the pen touching the paper for a signature, the technician should read the item before signing and confirm it has been performed.

\section{Lack of Knowledge}

A lack of knowledge when performing aircraft maintenance can result in a faulty repair that can have catastrophic results. Differences in technology from aircraft to aircraft and updates to technology and procedures on a single aircraft also make it challenging to have the knowledge required to perform airworthy maintenance.

All maintenance must be performed to standards specified in approved instructions. These instructions are based on knowledge gained from the engineering and operation of the aircraft equipment. Technicians must be sure to use the latest applicable data and follow each step of the procedure as outlined. They must also be aware that differences exist in the design and maintenance procedures on different aircraft. It is important for technicians to obtain training on different types of aircraft. When in doubt, a technician with experience on the aircraft should be consulted. If one is not available, or the consulted technician is not familiar with the procedure, a manufacturer' s technical representative should be contacted. It is better to delay a maintenance procedure than to do it incorrectly and cause an accident.

\section{Distraction}

A distraction while performing maintenance on an aircraft may disrupt the procedure. When work resumes, it is possible that the technician skips over a detail that needs attention. It is estimated that 15 percent of maintenance related errors are caused by distractions[4]. Distractions can be mental or physical in nature. They can occur when the work is located on the aircraft or in the hangar. They can also occur in the psyche of the technician independent of the work environment.
Something as simple as a cell phone call or a new aircraft being pushed into the hangar can disrupt the technician's concentration on a job. Less visible is a difficult family or financial matter or other personal issues that may occupy the technicians thought process as work is performed. This can make performance of the required maintenance less effective. Regardless of their nature, numerous distractions may occur during the course of maintaining an aircraft. The technician must recognize when attention to the job at hand is being diverted and assure that work continues correctly. A good practice is to go back three steps in the work procedure when one is distracted and resume the job from that point. Use of a detailed step-by-step written procedure and signing off each step only after it is completed also helps. Incomplete work can be marked or tagged, especially when the technician is pulled from the work by a distraction, and it is unknown when work will be resumed and by whom.

\section{E. Lack of Teamwork}

A lack of teamwork may also contribute to errors in aircraft maintenance. Closely related to lack of communication, teamwork is required in aviation maintenance in many instances. Sharing of knowledge between technicians, coordinating maintenance functions, turning work over from shift to shift, and working with flight personnel to troubleshoot and test aircraft are all are executed better in an atmosphere of teamwork. Often associated with improved safety in the workplace, teamwork involves everyone understanding and agreeing on actions to be taken. A gear swing or other operational check involves all the members of a team working together. Multiple technicians contribute to the effort to ensure a single outcome. They communicate and look out for one another as they do the job. A consensus is formed that the item is airworthy or not airworthy. The technician primarily deals with the physical aspect of the aircraft and its airworthiness. Others in the organization perform their roles and the entire company functions as a team. Teams can win or lose depending on how well everyone in the organization works together toward a common objective. A lack of teamwork makes all jobs more difficult and can result in a miscommunication that affects the airworthiness of the aircraft.

\section{F. Fatigue}

Fatigue is a major human factor that has contributed to many maintenance errors resulting in accidents[5]. Fatigue can be mental or physical in nature. Emotional fatigue also exists and effects mental and physical performance. A person is said to be fatigued when a reduction or impairment in any of the following occurs: cognitive ability, decision-making, reaction time, coordination, speed, strength, and balance. Fatigue reduces alertness and often reduces a person's ability to focus and hold attention on the task being performed. fatigue results in an increase in mistakes, poor judgment, and poor decisions or perhaps no decisions at all. A fatigued person may also lower his or her standards.

Tiredness is a symptom of fatigue. However, sometimes a fatigued person may feel wide awake and engaged in a task. The primary cause of fatigue is a lack of sleep. Good restful sleep, free from drugs or alcohol is a human necessity to prevent fatigue. Fatigue can also be caused by stress and 
overworking. A person's mental and physical state also naturally cycles through various levels of performance each day. Variables such as body temperature, blood pressure, heart rate, blood chemistry, alertness, and attention rise and fall in a pattern daily. This is known as one's circadian rhythm. A person's ability to work (and rest) rises and falls during this cycle. The best remedy for fatigue is to get enough sleep on a regular basis. The technician must be aware of the amount and quality of sleep obtained. Caution or time off is justified when too little sleep has occurred and errors are probable during maintenance.

Suggestions to help mitigate the problems caused by fatigue include looking for symptoms of fatigue in one's self and in others. Have others check your work, even if an inspector sign off is not required. Avoid complex tasks during the bottom of your circadian rhythm. Sleep and exercise daily. Eight to nine hours of daily sleep are recommended to avoid fatigue. AMTs in airline operations are part of a system in which most maintenance is performed at night. Fleet aircraft are operated primarily during the daytime hours to generate revenue. Therefore, shift work is required to maintain the fleet. It is already known that turning work over to other technicians during shift change is a problem that can lead to errors due to lack of communication. Shift work requires technicians to work during low cycles of their natural circadian rhythm. It also makes sleep more difficult when not on the job. Furthermore, regular night shift work makes one's body more sensitive to environmental disturbances. The technician must be aware that avoidance of fatigue is part of the job. it is up to organizations and technicians to regulate shift work and time off to reduce the potential for errors. Most importantly, each technician must monitor and control his or her sleep habits to avoid fatigue[6].

\section{G. Lack of Resources}

A lack of resources can interfere with one's ability to complete a task because there is a lack of supply and support. Low quality products also affect one's ability to complete a task. Aviation maintenance demands proper tools and parts to maintain a fleet of aircraft. Any lack of resources to safely carry out a maintenance task can cause both non-fatal and fatal accidents[4]. For example, if an aircraft is dispatched without a functioning system that is typically not needed for flight but suddenly becomes needed, this could create a problem.

Within an organization, making sure that personnel have the correct tools for the job is just as important as having the proper parts when they are needed. Having the correct tools means not having to improvise. For example, an aircraft that had received a new interior needed to be weighed prior to being released to fly. The aircraft was weighed without the proper electronic load cells placed between the aircraft jack and the aircraft. Then the aircraft slipped off of one of the load cells and the jack point creased the spar. The cost of improvising can be very steep. The right tools to do the job need to be used at all times, and if they are broken, out of calibration, or missing, they need to be repaired, calibrated, or returned as soon as possible.

Technical documentation is another critical resource that can lead to problems in aviation maintenance. Most manuals are in a constant state of revision and, if organizations do not identify missing information in the manuals, then nothing is done to correct the documentation. Resources, such as publication departments and manufacturer's technical support, are available and should be used rather than ignoring the problem.

Another valuable resource that the maintenance department should rely on is the flight crew. Organizations should encourage open communication between the flight crews and the maintenance crews. The flight crew can provide valuable information when dealing with a defective part or problem.

\section{H. Pressure}

Aviation maintenance tasks require individuals to perform in an environment with constant pressure to do things better and faster without making mistakes and letting things fall through the cracks. Unfortunately, these types of job pressures can affect the capabilities of maintenance workers to get the job done right. Airlines have strict financial guidelines, as well as tight flight schedules, that force mechanics to be under pressure to identify and repair mechanical problems quickly so that the airline industry can keep moving. Most important, aircraft mechanics are responsible for the overall safety of everyone who uses flying as a mode of transportation.

Organizations must be aware of the time pressures that are put on aircraft mechanics and help them manage all of the tasks that need to be completed so that all repairs, while done in a timely manner, are completed correctly with safety being the ultimate goal. Sacrificing quality and safety for the sake of time should not be tolerated or accepted. Likewise, AMTs need to recognize on their own when time pressures are clouding their judgments and causing them to make unnecessary mistakes. Self-induced pressures are those occasions where one takes ownership of a situation that was not of their doing.

In an effort to combat self-induced pressure, technicians should ask for help if they feel overwhelmed and under a time constraint to get a repair fixed. Another method is to have someone check the repair thoroughly to ensure that all maintenance tasks were completed correctly. Lastly, if given a repair with a specific time limitation that you do not feel is realistic or compromises safety, bring it to the attention of the organization's management and openly discuss a different course of action.

\section{Lack of Assertiveness}

Assertiveness is the ability to express your feelings, opinions, beliefs, and needs in a positive, productive manner and should not be confused with being aggressive. It is important for AMTs to be assertive when it pertains to aviation repair rather than choosing or not being allowed to voice their concerns and opinions. The direct result of not being assertive could ultimately cost people their lives.

When being assertive with co-workers or management, deal with one issue at a time rather than trying to tackle a number of problems at once. It is also important to have documentation and facts to back up your argument, which can give people a visual account of what you are trying to explain. A lack of assertiveness in failing to speak up when things do not seem right has resulted in many fatal accidents. This can easily be 
changed by promoting good communication between co-workers and having an open relationship with supervisors and management. Maintenance managers must be familiar with the behavior style of the people they supervise and learn to utilize their talents, experience, and wisdom. AMTs should give supervisors and management the kind of feedback required to ensure that they will be able to assist the mechanic to do their job.

\section{J. Stress}

Aviation maintenance is a stressful task due to many factors. Aircraft must be functional and flying in order for airlines to make money, which means that maintenance must be done within a short timeframe to avoid flight delays and cancellations. Fast-paced technology that is always changing can add stress to technicians. This demands that AMTs stay trained on the latest equipment. Other stressors include working in dark, tight spaces, lack of resources to get the repair done correctly, and long hours. The ultimate stress of aviation maintenance knows that the work they do, if not done correctly, could result in tragedy. Everyone handles stress differently and particular situations can bring about different degrees of difficulty for different people. For example, working under a strict timeline can be a stressor for one person and normal for another. The causes of stress are referred to as stressors. They are categorized as physical, psychological, and physiological stressors. Following is a list of each and how they may affect maintenance.

\section{1) Physical Stressors}

Physical stressors add to the personnel's workload and make it uncomfortable for him or her in their work environment.

- Temperature-high temperatures in the hanger increases perspiration and heart rate causing the body to overheat. Low temperatures can cause the body to feel cold, weak, and drowsy.

Noise-hangers that have high noise levels (due to aircraft taking off and landing close by) can make it difficult for maintenance personnel to focus and concentrate.

- Lighting-poor lighting within a work space makes it difficult to read technical data and manuals. Likewise, working inside an aircraft with poor lighting increases the propensity to miss something or to repair something incorrectly.

Confined spaces-small work spaces make it very difficult to perform tasks as technicians are often contorted into unusual positions for a long period of time.

\section{2) Psychological Stressors}

Psychological stressors relate to emotional factors, such as a death or illness in the family, business worries, poor interpersonal relationships with family, co-workers, supervisors, and financial worries.

- Work-related stressors-over anxiousness can hinder performance and speed while conducting maintenance if there is any apprehension about how to do a repair or concerns about getting it done on time.
- Financial problems-impending bankruptcy, recession, loans, and mortgages are a few examples of financial problems that can create stressors.

- Marital problems-divorce and strained relationships can interfere with one's ability to perform their job correctly.

- Interpersonal problems-problems with superiors and colleagues due to miscommunication or perceived competition and backstabbing can cause a hostile work environment.

\section{3) Physiological Stressors}

Physiological stressors include fatigue, poor physical condition, hunger, and disease.

- Poor physical condition-trying to work when ill or not feeling well can force the body to use more energy fighting the illness and less energy to perform vital tasks.

- Proper meals-not eating enough, or foods lacking the proper nutrition, can result in low energy and induce symptoms like headaches and shaking.

- Lack of sleep-fatigued, the maintainer is unable to perform to standard for long periods of time and can become sloppy with repairs and miss important mistakes.

- Conflicting shift schedules-the effect of changing sleep patterns on the body's circadian cycle can lead to a degradation of performance.

People cope with stress in many different ways. Specialists say that the first step is to identify stressors and the symptoms that occur after exposure to those stressors. Other recommendations involve development or maintenance of a healthy lifestyle with adequate rest and exercise, a healthy diet, limited consumption of alcoholic drinks, and avoidance of tobacco products.

\section{K. Lack of Awareness}

Lack of awareness is defined as a failure to recognize all the consequences of an action or lack of foresight. In aviation maintenance, it is not unusual to perform the same maintenance tasks repeatedly. After completing the same task multiple times, it is easy for technicians to become less vigilant and develop a lack of awareness for what they are doing and what is around them. Each time a task is completed it must be treated as if it were the first time.

\section{Norms}

Norms is short for "normal" or the way things are normally done. They are unwritten rules that are followed or tolerated by most organizations[2]. Negative norms can detract from the established safety standard and cause an accident to occur. Norms are usually developed to solve problems that have ambiguous solutions. When faced with an ambiguous situation, an individual may use another's behavior as a frame of reference around which to form his or her own reactions. As this process continues, group norms develop and stabilize. Newcomers to the situation are then accepted into the group based on adherence to norms. Very rarely do newcomers initiate change in a group with established norms.

Some norms are unsafe in that they are non-productive or 
detract from the productivity of the group. Taking shortcuts in aircraft maintenance, working from memory, or not following procedures are examples of unsafe norms. Newcomers are better able to identify these unsafe norms than long-standing members of the group. On the other hand, the newcomer's credibility depends on his or her assimilation into the group. The newcomer's assimilation, however, depends on adherence to the group norms. Everyone should be aware of the perceptiveness of newcomers in identifying unhealthy norms and develop a positive attitude toward the possibility that norms may need to be changed. Finally, as newcomers become assimilated into the group structure, they build credibility with others. Once this has been done, a relative newcomer may begin to institute change within the group. Unfortunately, such actions are often difficult to do and rely heavily on the group's perception of the newcomer's credibility.

Norms have been identified as one of the human factors in aviation maintenance and a great deal of anecdotal evidence points to the use of unsafe norms on the line. The effect of unsafe norms may range from the relatively benign, such as determining accepted meeting times, to the inherently unsafe, such as signing off on incomplete maintenance tasks. Any behavior commonly accepted by the group, whether as a standard operating procedure (SOP) or not, can be a norm.

Supervisors need to ensure that everyone adheres to the same standards and not tolerate unsafe norms. AMTs should pride themselves on following procedure, rather than unsafe norms that may have been adopted as regular practice.

\section{$\mathrm{V}$ CONCLUSIONS}

By understanding each of the disciplines and applying them to different situations or human behaviors, we can correctly recognize potential human factors and address them before they develop into a problem or create a chain of problems that result in an accident or incident. Human factors awareness can lead to improved quality, an environment that ensures continuing worker and aircraft safety, and a more involved and responsible work force. More specifically, the reduction of even minor errors can provide measurable benefits including cost reductions, fewer missed deadlines, reduction in work related injuries, reduction of warranty claims, and reduction in more significant events that can be traced back to maintenance error.

\section{REFERENCES}

[1] Allen, Jr., J.A., Rankin, W.L., 1995. A Summary of the Use and Impact of the Maintenance Error Decision Aid (MEDA) on the Commercial Aviation Industry. Proceedings of the Flight Safety Foundation 48th Annual International Air Safety Seminar, International Federation of Airworthiness 25th International Conference, and the International Air Transport Association \&Managing Safety', November 7\}9, Seattle,WA, USA.

[2] Reason, J., 1990. Human Error. Cambridge University Press, New York.

[3] Graeber, R.C. \& Marx, D.A. (1993). Reducing human error in aircraft ma intenance operations. Paper presented at the Flight Safety Foundation 46th Annual International Air Safety Seminar, Kuala Lumpur, Malaysia.

[4] Hobbs, A. \& Williamson, A. (2001). Aircraft Maintenance Safety Survey-Results Department of Transport and Regional Services, Australian Transport Safety Bureau.

[5] Federal Aviation Administration (2011). Fatigue Risk Management in Aviation Maintenance: Current Best Practices and Potential Future Countermeasures

[6] Federal Aviation Administration (2013). An Evaluation of Aviation Maintenance Fatigue Countermeasures Training

[7] National Transportation Safety Board (2001b). Accidents, fatalities, and rates, 1982 through 2000, for US air carriers operating under 14 CFR 121 , scheduled and nonscheduled service. 